\title{
Formación de profesores universitarios en posgrados interdisciplinarios. Dificultades en la escritura del plan de tesis
}

\author{
María Isabel Pozzo*1; Thalita Camargo Angelucci²; Ana Luisa Cardoso ${ }^{3}$ \\ ${ }^{1}$ https://orcid.org/0000-0003-0186-0910, Instituto Rosario de Investigaciones en Ciencias de la Educación, Consejo Nacional de \\ Investigaciones Científicas y Técnicas, Argentina, ${ }^{2}$ https://orcid.org/0000-0002-0158-7953, ${ }^{3}$ https://orcid.org/0000-0003-3365- \\ 6201, 2, 3 Instituto Rosario de Investigaciones en Ciencias de la Educación, Consejo Nacional de Investigaciones Científicas y \\ Técnicas, Argentina
}

Citar como: Pozzo, M.I., Camargo Angelucci, T, \& Cardoso, A. L. (2021). Formación de profesores universitarios en posgrados interdisciplinarios. Dificultades en la escritura del plan de tesis. Revista Digital de Investigación en Docencia Universitaria, 15(2), e1238. https://doi.org/10.19083/10.19083/ridu.2021.1238

Recibido: 23/06/2020. Revisado: 15/10/2020. Aceptado: 01/11/2021. Publicado: 26/11/2021.

\begin{abstract}
Resumen
Introducción: Los estudios de posgrado permiten a los graduados universitarios continuar su desarrollo profesional, incluso en áreas disciplinares diferentes a las de sus estudios previos. A tal fin, los posgrados exigen a los cursantes la elaboración de un Plan de tesis previo a la realización de una tesis, requisito central de este nivel educativo. Estos requerimientos suponen conocimientos que no siempre se proporcionan en las diversas carreras de grado. Objetivo: En este contexto, se presenta un análisis de las principales dificultades de escritura identificadas en Planes de Tesis elaborados por profesores universitarios provenientes de diferentes áreas del saber en el marco de una maestría de formación en docencia universitaria de Argentina. Método: Para ello, se compilan 13 Planes de tesis presentados para aprobar el Seminario final adoptados como unidades de análisis. Se realiza una corrección exhaustiva de cada Plan y a continuación, las dificultades encontradas son categorizadas en microestructurales, macroestructurales y superestructurales (Van Dijk, 1980) y subcategorizadas. Resultados: Los resultados dan cuenta de una cantidad y variedad importante de dificultades en las tres categorías en todos los cursantes. Conclusión: De esta cantidad y variedad de dificultades se concluye la necesidad de diseñar e implementar prácticas de enseñanza integrales para culminar la tesis.
\end{abstract}

Palabras clave: escritura; posgrado; dificultad de aprendizaje; ciencias de la educación.

\section{Training of university teachers in interdisciplinary postgraduate courses. Difficulties in writing the Thesis Plan}

\footnotetext{
Abstract

Introduction: Postgraduate studies allow university graduates to continue their professional development, even in disciplines different from their previous studies. To this end, postgraduate courses require students to prepare a thesis plan as a prior step to completing a thesis, a central requirement of this educational level. These requirements involve
} 
knowledge that is not always provided in the various graduate courses. Objective: In this context, in this paper we present an analysis of the main writing difficulties identified in Thesis Plans written by university teachers from different areas of knowledge within the framework of a master's degree in university teaching training in Argentina. Method: For this, 13 thesis plans presented to pass the final Master's Seminar are compiled and adopted as units of analysis. An exhaustive correction of each Plan is made; then, the difficulties encountered are categorized into microstructural, macrostructural and superstructural (Van Dijk, 1980) and in turn subcategorized. Results: The results show a significant amount and variety of difficulties in the three categories in all the students. Conclusion: From the amount and variety of difficulties found, the need to design and implement comprehensive teaching practices to complete the thesis is concluded.

Keywords: writing; postgraduate; learning difficulties; educational sciences.

\section{Introducción}

La escritura en la universidad se caracteriza por la producción de géneros discursivos de carácter científico y profesional, como pueden ser los informes, artículos, monografías, tesis, entre otros (Swales, 2004). Se entiende por géneros discursivos aquellos tipos relativamente estables de enunciados que circulan en esferas sociales de la comunicación verbal (Bajtín, 2002). Asimismo, se conciben los textos como producciones verbales empíricamente realizadas en distintos formatos que varían según las particularidades de la situación de comunicación en que se producen (Bronckart, 2004). De ese modo, en cada producción comunicativa social se espera la articulación de tres aristas: el contenido temático, el estilo verbal y la composición.

En ese sentido, los textos académicos también siguen cánones en su desarrollo. En el caso de la formación de posgrado, para la obtención de los títulos de magíster y doctor, se espera como producto final una tesis escrita y defendida. Para esto, los tesistas necesitan plantear un texto estructurado en forma apropiada, según las pautas de una determinada comunidad discursiva (Swales, 1990). Ahora bien, el paso previo y fundamental a la escritura de la tesis es la redacción de un Plan de Tesis que también sigue patrones específicos. En el género en cuestión, se espera: una introducción que presente contextualizadamente el problema de investigación y los objetivos; una revisión de antecedentes y el encuadre teórico, el método seleccionado y un cronograma de actividades. Si bien la meta resulta apropiada, su concreción resulta un verdadero desafío para sus actores y ha sido motivo de numerosas reflexiones (Arnoux, 2009; Carlino, 2013).

En una revisión de la literatura en lengua castellana sobre la escritura en posgrados, Chois y Jaramillo (2016) observaron una mayoría de estudios realizados en Argentina respecto de otros países. Los autores advirtieron que 32 de los 39 proyectos analizados se abocaban a la producción escrita de los estudiantes. Además, ubicaron los problemas de escritura y lectura de los estudiantes entre los de mayor frecuencia, al igual que otros estudios sobre el posgrado (Pozzo, 2019; Borioli, 2019).

Otros antecedentes acerca de la escritura académica (Arroyo González \& Gutierrez Braojos, 2016; Castro Azuara \& Sánchez Camargo, 2016; Coronado-Hijón, 2017; Núñez Cortés \& Errázuriz Cruz, 2020) han desvelado carencias por universitarios. Las dificultades se enmarcan tanto en los ámbitos de dominio formal, estructural y discursivo como en el del rol de autor-investigador (carencia de la voz autoral).

Se parte de la literatura que trata de la escritura académica en la universidad en general (Cassany, 2009; Klein, 2007; Schere, 2020) y en el posgrado en particular (Eco, 1998; Giraldo-Giraldo, 2020; Nishida, 2020; Mendoza Ramos, 2014; Rodríguez Hernández \& García Valero, 2015), y el interaccionismo socio discursivo para caracterizar al referido género textual (Bronckart, 2004).

Por otra parte, se identificaron tres grandes categorías como criterio apropiado para el análisis de producciones escritas (Fuenmayor, Villasmil \& Rincón, 2008): dificultades de microestructura, de macroestructura y de superestructura (Van Dijk, 1980). 
La Microestructura corresponde al léxico, a la gramática y a la estructura de las proposiciones de un texto, por lo tanto, es de carácter local. Se conforma a partir del conjunto de mecanismos que permite dar linealidad al texto y conectar sus distintas partes (sintagmas, oraciones, párrafos, fragmentos) entre sí y con el contexto. Comprende elementos de cohesión (puntuación, conectores, marcadores discursivos y relaciones verbales) que permiten la articulación entre ideas. Atiende a la idiosincrasia de la gramática de la lengua en la que se produce y de la semántica en su sentido superficial (Cassany, 2009; Ilich \& Morales, 2004; Van Dijk, 1980).

La Macroestructura se refiere a la estructura más global del texto, es decir, representa un encadenamiento lógico de ideas observable a partir de la identificación del tema tratado y su progresión temática. Se materializa especialmente en la coherencia entre párrafos y de estos con la idea central planteada en el texto (Ilich \& Morales, 2004; Rueda, 2007; Van Dijk, 1980). Además, es de carácter básicamente pragmático y semántico -en su sentido profundo- (Cassany, 2009).

En lo concerniente a la Superestructura, allí se enmarca la adecuación genérica del texto. En este sentido, se consideran los problemas de convenciones de estilo y presentación gráfica: márgenes, fuentes, interlineado, etc. (Van Dijk, 1980).

Partiendo de este contexto, el objetivo del presente trabajo es identificar las dificultades en la escritura del género discursivo Plan de Tesis considerando los tres grandes parámetros descriptos. De estos textos se espera un plan de investigación factible y riguroso para un posgrado interdisciplinario; específicamente, la Maestría en Docencia Universitaria de una universidad pública argentina (Gioffredo y Pozzo, 2015). De estos textos se espera que informen un plan de investigación factible y riguroso, cuyo ámbito académico primario de circulación es el Seminario que se propone acreditar y la facultad donde se dicta la carrera de posgrado, destinado asimismo a la comunidad científica. A partir de la caracterización de las dificultades de los estudiantes de posgrado se espera aportar estrategias de escritura más eficaces (Pozzo, 2020).

\section{Método}

\section{Diseño}

Este estudio se enmarca metodológicamente en un estudio de caso, abordado desde una perspectiva descriptiva (Stake, 1998). En esta línea, el caso corresponde al Seminario de tesis, dictado por la primera autora de esta publicación, en el marco de la Maestría en Docencia Universitaria de la Universidad Tecnológica Nacional de Argentina, en una de sus Facultades Regionales. Este Seminario es el auxilio más cercano en temas de escritura referida a la investigación, ya que no existe en la currícula un seminario específico destinado a tal fin. Esta vacancia coincide con lo observado en otras carreras de posgrado (Coronado-Hijón, 2017; Nishida, 2020). Este hecho da cuenta de que se asume que un profesional graduado tiene resuelta la práctica escrituraria, o bien tiene dificultades leves. El trabajo parte del análisis de un corpus textual compuesto por 13 planes de tesis recolectados en diciembre del 2019, con una cohorte completa.

\section{Unidades de análisis}

Las unidades de análisis del presente estudio son, como se dijo, los Planes de tesis. Sus autores son docentes de diferentes carreras universitarias; siete tienen entre 30 y 40 años y la otra mitad, entre 40 y 60. Son graduados/as de las siguientes carreras, en las cantidades indicadas: Enfermería (2), Medicina (2), Ingeniería Química (1), Ingeniería Civil (1), Veterinaria (3), Fonoaudiología (1), Kinesiología (1), Marketing (1) y Sociología (1). Como puede advertirse, se trató de un grupo bastante heterogéneo en cuanto a sus especialidades. Tal multiplicidad de disciplinas es una de las características de esta Maestría, ya que funciona como formación docente de profesionales de distintas áreas.

Los planes de tesis fueron producidos siguiendo el modelo de la universidad correspondiente, que consta de las siguientes secciones: Justificación del tema elegido, Fundamentación teórica, Objetivos, Método de recolección de información, Factibilidad, Cronograma y Referencias bibliográficas. Como se aprecia, responde a lo convencionalmente establecido (Pozzo, 2020). Los planes fueron presentados a la docente a cargo del 
seminario mencionado para la aprobación del mismo. Asimismo, se convierte en un insumo para ser evaluados a nivel nacional para su aceptación formal como cursante de Maestría.

\section{Procedimiento}

Para proceder a los análisis se codificó cada texto, cuya área de formación de su autor consta en la Tabla 1. Esta información se expone a modo de referencias, ya que la relación entre las dificultades en la escritura y el área de formación académica no representa nuestro objetivo.

En primer lugar, se realizó una exhaustiva corrección textual de los planes de tesis en cuanto a las inadecuaciones lingüísticas, discursivas y de convenciones académicas propias del género. El siguiente paso fue analizar los errores y categorizarlos. A partir del análisis se establecieron tres grandes áreas de dificultades presentadas por los cursantes en sus textos: de microestructura, de macroestructura y de superestructura, detallando en cada caso el tipo de error presentado.

\section{Análisis de datos}

Se informaron de las dificultades recabadas disponiendo, además, la frecuencia de sus ocurrencias en los textos escritos para una visión general de la problemática (Tabla 2).

Con el objeto de lograr una mejor interpretación de los datos, se realizó un registro descriptivo de las dificultades más recurrentes a las que los alumnos se enfrentan al redactar un Plan de tesis. A lo largo del análisis, se seleccionaron fragmentos que ejemplifican las discusiones llevadas a cabo. Estos fragmentos fueron reproducidos tal como aparecen en los textos del corpus, identificados por su respectivo número.

\section{Resultados y Discusión}

A partir de las correcciones realizadas en los textos, se identificaron las principales dificultades de escritura de los cursantes y se clasificaron dentro de tres grandes categorías y subcategorías. De ese modo, se obtuvo un total de 64 ítems (Tablas 2, 3 y 4).

Tabla 1.

Código de los textos y carrera de los cursantes-autores de los textos

\begin{tabular}{ll}
\hline Código del texto & Carrera del cursante-autor del texto \\
\hline 1 & Marketing \\
2 & Enfermería \\
3 & Medicina \\
4 & Ingeniería Química \\
5 & Kinesiología y Fisiatría \\
6 & Ingeniería Civil \\
7 & Enfermería \\
8 & Medicina \\
9 & Fonoaudiología \\
10 & Veterinaria \\
11 & Sociología \\
12 & Veterinaria \\
13 & Veterinaria \\
\hline
\end{tabular}


Tabla 2.

Dificultades de microestructura

\begin{tabular}{|c|c|c|c|c|c|c|c|c|c|c|c|c|c|c|c|c|}
\hline \multirow{2}{*}{ Categorías } & \multirow{2}{*}{ Subcategorías } & \multicolumn{13}{|c|}{ Código de texto } & \multirow[t]{2}{*}{ Total } & \multirow[t]{2}{*}{$\%$} \\
\hline & & 1 & 2 & 3 & 4 & 5 & 6 & 7 & 8 & 9 & 10 & 11 & 12 & 13 & & \\
\hline \multirow{7}{*}{ 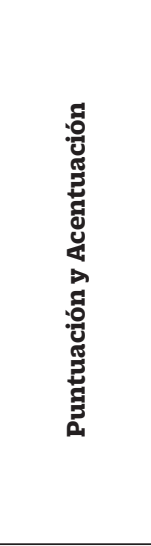 } & $\begin{array}{l}\text { Coma: omisión; empleo } \\
\text { inadecuado. }\end{array}$ & & 1 & 5 & 1 & 6 & 1 & 3 & 3 & 3 & 4 & 1 & 8 & 1 & 45 & 32,1 \\
\hline & Dos puntos: omisión. & 3 & 1 & 1 & 1 & & & 1 & 1 & 1 & 1 & & 1 & & 11 & 7,9 \\
\hline & $\begin{array}{l}\text { Punto y coma: ausencia; empleo } \\
\text { inadecuado. }\end{array}$ & & & & & 1 & & 1 & 1 & 3 & & & & 1 & 7 & 5,0 \\
\hline & $\begin{array}{l}\text { Tilde: omisión; empleo } \\
\text { inadecuado. }\end{array}$ & & 3 & 1 & 1 & 1 & 1 & 3 & 2 & 8 & 2 & 1 & 6 & & 29 & 20,7 \\
\hline & Paréntesis: empleo innecesario. & & & & 1 & & & & & 1 & & & & & 2 & 1,4 \\
\hline & Comillas: empleo innecesario. & & & & & & 1 & & & & & & & & 1 & 0,7 \\
\hline & $\begin{array}{l}\text { Puntos suspensivos: empleo } \\
\text { innecesario. }\end{array}$ & & & & & & & & & & & & 1 & & 1 & 0,7 \\
\hline \multirow{11}{*}{$\stackrel{\circ}{\text { 营 }}$} & Mayúsculas: empleo innecesario & 1 & & & & & & & & 2 & 1 & 1 & & & 5 & 3,6 \\
\hline & Mayúsculas: omisión. & & 1 & & & & & & & & & & & & 1 & 0,7 \\
\hline & Conectores oracionales: omisión. & & & & & & 1 & & & & 1 & & 4 & & 6 & 4,3 \\
\hline & $\begin{array}{l}\text { Conectores oracionales: empleo } \\
\text { innecesario o inadecuado. }\end{array}$ & & & & & 1 & & & & & & 1 & 3 & & 5 & 3,6 \\
\hline & Oraciones truncas. & & 1 & 1 & & 3 & & & & & & & 1 & & 6 & 4,3 \\
\hline & $\begin{array}{l}\text { Construcción de los objetivos sin el } \\
\text { empleo de verbos en infinitivo. }\end{array}$ & & 1 & & & & & & & & & & & & 1 & 0,7 \\
\hline & $\begin{array}{l}\text { Repetición muy cercana de un } \\
\text { mismo término. }\end{array}$ & & & 2 & & & & 2 & & & & & & & 4 & 2,9 \\
\hline & Empleo de lenguaje coloquial. & & & 2 & 1 & 1 & & 2 & 1 & & & & 3 & & 10 & 7,1 \\
\hline & Falta de correlatividad verbal. & & & 1 & & & & & & & 1 & & & & 2 & 1,4 \\
\hline & $\begin{array}{l}\text { Uso inadecuado de pronombre } \\
\text { relativo. }\end{array}$ & & & & & & & & & 1 & & & & & 1 & 0,7 \\
\hline & $\begin{array}{l}\text { Ausencia de concordancia nominal } \\
\text { o verbal. }\end{array}$ & & & 2 & & & 1 & & & & & & & & 3 & 2,1 \\
\hline & TOTAL & & & & & & & & & & & & & & 140 & $100 \%$ \\
\hline
\end{tabular}


Tabla 3.

Dificultades de macroestructura

\begin{tabular}{|c|c|c|c|c|c|c|c|c|c|c|c|c|c|c|c|c|}
\hline \multirow{2}{*}{ Categorías } & \multirow{2}{*}{ Subcategorías } & \multicolumn{13}{|c|}{ Código de texto } & \multirow[t]{2}{*}{ Total } & \multirow[t]{2}{*}{$\%$} \\
\hline & & 1 & 2 & 3 & 4 & 5 & 6 & 7 & 8 & 9 & 10 & $\mathbf{1 1}$ & 12 & 13 & & \\
\hline \multirow{22}{*}{ 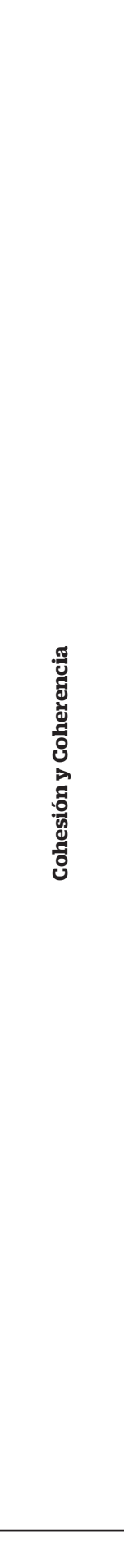 } & $\begin{array}{l}\text { Incoherencia entre ideas y/o } \\
\text { objetivos (y entre párrafos). }\end{array}$ & 3 & 1 & 2 & 5 & 1 & 1 & 2 & 2 & 2 & 1 & 1 & 1 & 2 & 24 & 18,6 \\
\hline & $\begin{array}{l}\text { Falta de cohesión entre el cierre de } \\
\text { una sección e inicio de otra o entre } \\
\text { párrafos. }\end{array}$ & & & & 1 & 1 & 1 & & 1 & & 2 & & & & 6 & 4,7 \\
\hline & Información incompleta/imprecisa. & 3 & 1 & 1 & 11 & 2 & 4 & 4 & & 2 & 3 & 3 & 5 & 2 & 41 & 31,8 \\
\hline & Secciones incompletas. & & & & 1 & & & & & & & 1 & & & 2 & 1,6 \\
\hline & Oraciones demasiado largas. & 1 & 1 & & & & & & 1 & & 2 & & & & 5 & 3,9 \\
\hline & Oraciones demasiado cortas. & & & & & & & 2 & 1 & 1 & 2 & & & & 6 & 4,7 \\
\hline & Párrafos demasiado cortos. & & & & & 1 & & & & & & & & & $\mathbf{1}$ & 0,8 \\
\hline & Repetición de sintagmas/ideas. & & & 1 & & 1 & & & & & & & & & 2 & 1,6 \\
\hline & $\begin{array}{l}\text { Temas distintos en un mismo } \\
\text { párrafo o frase. }\end{array}$ & & 1 & 1 & 1 & & & & & & & & & & 3 & 2,3 \\
\hline & $\begin{array}{l}\text { División inadecuada de párrafo } \\
\text { (respecto al tema). }\end{array}$ & & & & & 2 & & & 1 & & & 2 & & & 5 & 3,9 \\
\hline & $\begin{array}{l}\text { Ausencia de marcadores discursivos } \\
\text { que indican el cambio de ideas } \\
\text { entre párrafos. }\end{array}$ & & 1 & & & & & & 1 & & 1 & & & & 3 & 2,3 \\
\hline & $\begin{array}{l}\text { Ausencia de marcador discursivo } \\
\text { que indique el inicio de una oración. }\end{array}$ & 1 & & & & & & & & & & & & & 1 & 0,8 \\
\hline & $\begin{array}{l}\text { Uso innecesario de marcador } \\
\text { discursivo. }\end{array}$ & & 2 & & & & & & & & & & & & 2 & 1,6 \\
\hline & $\begin{array}{l}\text { Usos inadecuados de términos de } \\
\text { personalización. }\end{array}$ & & & 1 & 1 & & & & & & & & & & 2 & 1,6 \\
\hline & Ausencia de lenguaje contundente. & & & 1 & & & & & 1 & & 3 & & 4 & 1 & 10 & 7,8 \\
\hline & $\begin{array}{l}\text { Empleo inadecuado de lenguaje } \\
\text { contundente. }\end{array}$ & & & & 1 & & & & & & & & & & 1 & 0,8 \\
\hline & $\begin{array}{l}\text { Uso inadecuado de términos que } \\
\text { denotan imposición/moralismo. }\end{array}$ & & & & 1 & & & & & & & & & & 1 & 0,8 \\
\hline & $\begin{array}{l}\text { Temas ubicados en secciones con } \\
\text { las que no se corresponden. }\end{array}$ & 2 & & & 1 & & & & & 1 & 1 & & & 2 & 7 & 5,4 \\
\hline & $\begin{array}{l}\text { Uso de referencias bibliográficas no } \\
\text { correspondientes al tema. }\end{array}$ & & & & 1 & & & & & & & & & & 1 & 0,8 \\
\hline & $\begin{array}{l}\text { Ausencia de foco en la } \\
\text { problematización (hay varias } \\
\text { preguntas). }\end{array}$ & & & & & & 1 & & & & & & & & 1 & 0,8 \\
\hline & $\begin{array}{l}\text { Utilización de palabras diferentes } \\
\text { como equivalentes (ejemplo: } \\
\text { "escritura" y "lectoescritura"). }\end{array}$ & & & 2 & 3 & & & & & & & & & & 5 & 3,9 \\
\hline & TOTAL & & & & & & & & & & & & & & 129 & $100 \%$ \\
\hline
\end{tabular}


Tabla 4.

Dificultades de superestructura

\begin{tabular}{|c|c|c|c|c|c|c|c|c|c|c|c|c|c|c|c|c|}
\hline \multirow{2}{*}{ Categorias } & \multirow{2}{*}{ Subcategorías } & \multicolumn{13}{|c|}{ Código de texto } & \multirow[t]{2}{*}{ Total } & \multirow[t]{2}{*}{$\%$} \\
\hline & & 1 & 2 & 3 & 4 & 5 & 6 & 7 & 8 & 9 & 10 & 11 & 12 & 13 & & \\
\hline \multirow{7}{*}{ 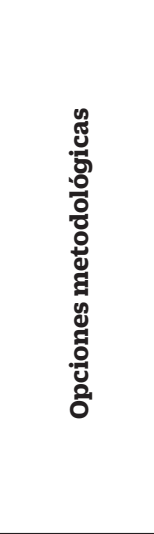 } & $\begin{array}{l}\text { Incoherencia en la clasificación del tipo de } \\
\text { investigación. }\end{array}$ & 1 & & & 1 & & & & & & & & & & 2 & 1,9 \\
\hline & $\begin{array}{l}\text { Ausencia de definición del tipo de } \\
\text { investigación. }\end{array}$ & & & 1 & & & 1 & & & & & & & & 2 & 1,9 \\
\hline & $\begin{array}{l}\text { Ausencia de informaciones acerca de los } \\
\text { procedimientos metodológicos. }\end{array}$ & & & & 1 & 1 & & & & & & & & & 2 & 1,9 \\
\hline & $\begin{array}{l}\text { Debilidad en la justificación del foco del } \\
\text { trabajo. }\end{array}$ & 1 & & & & & & & & 1 & 1 & & & 1 & 4 & 3,8 \\
\hline & Ausencia de presentación del tipo de muestra. & & & & 1 & & & 1 & & & & & & & 2 & 1,9 \\
\hline & $\begin{array}{l}\text { Número de informantes elegido para el } \\
\text { estudio no indicado o no justificado }\end{array}$ & & 1 & & & 1 & & & & & & & & & 2 & 1,9 \\
\hline & Ausencia de objetivos específicos. & & & & & & 1 & & & & & & & & 1 & 0,9 \\
\hline \multirow{14}{*}{ 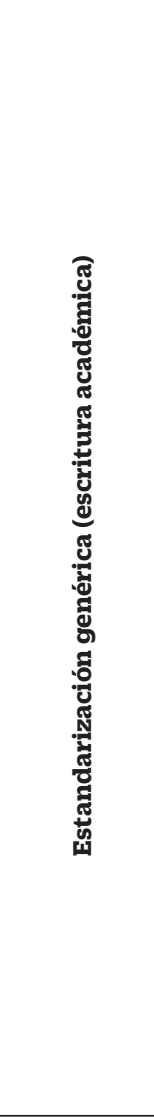 } & Omisión de número de páginas en las citas. & 1 & & & 1 & & & 1 & 1 & 1 & & & & & 5 & 4,7 \\
\hline & $\begin{array}{l}\text { Empleo inadecuado de comillas en citas } \\
\text { largas. }\end{array}$ & & & 1 & & & 1 & & & 2 & 1 & & & 1 & 6 & 5,7 \\
\hline & $\begin{array}{l}\text { Omisión de paréntesis donde habría que } \\
\text { presentarlos. }\end{array}$ & 3 & & & & & & & & & & & & & 3 & 2,8 \\
\hline & $\begin{array}{l}\text { Empleo innecesario e inadecuado de iniciales } \\
\text { de nombres de autores en medio al texto. }\end{array}$ & & 1 & & & & & & & & & & & & 1 & 0,9 \\
\hline & $\begin{array}{l}\text { Empleo repetido de apellidos de autores en } \\
\text { medio al texto. }\end{array}$ & & & & 3 & & & & & & & & & & 3 & 2,8 \\
\hline & $\begin{array}{l}\text { Mezcla de sistemas de estandarización de } \\
\text { escritura científica a lo largo del trabajo. }\end{array}$ & & & 1 & & 1 & & & & & & & & & 2 & 1,9 \\
\hline & Referencias bibliográficas incompletas & & & 4 & 4 & 3 & & 2 & 1 & 4 & 1 & 2 & 1 & 1 & 23 & 21,7 \\
\hline & $\begin{array}{l}\text { Errores en la estandarización de las } \\
\text { "Referencias bibliográficas". }\end{array}$ & 3 & 2 & & 2 & 1 & & 1 & 3 & 5 & 4 & 1 & & 1 & 23 & 21,7 \\
\hline & Inadecuación de márgenes. & & & & & 1 & & & & & & & & & 1 & 0,9 \\
\hline & $\begin{array}{l}\text { Omisión de aclaración de siglas o uso } \\
\text { inadecuado de siglas. }\end{array}$ & & & 1 & & & 1 & & 2 & & & & & & 4 & 3,8 \\
\hline & $\begin{array}{l}\text { Presentación de citas de documentos } \\
\text { sin referencia a la institución autora del } \\
\text { documento. }\end{array}$ & & & & & & 1 & & & & & & & & 1 & 0,9 \\
\hline & Ausencia de la sección "Referencias". & & & & & & 1 & & & & & & & & 1 & 0,9 \\
\hline & $\begin{array}{l}\text { Empleo de secciones fuera del orden secuencial } \\
\text { adecuado al género Proyecto de Tesis. }\end{array}$ & & & & & & 1 & & & & & & & 1 & 2 & 1,9 \\
\hline & $\begin{array}{l}\text { Metalenguaje: ausencia de recursos que } \\
\text { distingan el metalenguaje. }\end{array}$ & & & 1 & & & & & & & & & 1 & & 2 & 1,9 \\
\hline \multirow{5}{*}{ 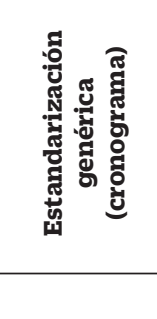 } & $\begin{array}{l}\text { Incoherencia en la división del tiempo de las } \\
\text { actividades del cronograma. }\end{array}$ & 1 & & & & & & & 1 & & & & & & 2 & 1,9 \\
\hline & $\begin{array}{l}\text { Incoherencia en el orden de las actividades del } \\
\text { cronograma. }\end{array}$ & & 2 & 1 & & & & 1 & 2 & 1 & & & 1 & & 8 & 7,5 \\
\hline & Enumeración de actividades repetidas. & & & & & & & 3 & & & & & & & 3 & 2,8 \\
\hline & Ausencia de cronograma & & & & & & & & & & & 1 & & & 1 & 0,9 \\
\hline & TOTAL & & & & & & & & & & & & & & 106 & $100 \%$ \\
\hline
\end{tabular}


En los siguientes apartados se caracterizan y discuten fragmentos del corpus que representan las dificultades más recurrentes de los cursantes. En algunos casos, también se proponen posibles resoluciones.

\section{Dificultades de microestructura}

Las dificultades de microestructura se refieren a las ocurrencias dentro los límites de la oración. Los problemas más relevantes desde esta perspectiva, por un lado, se advierten en el uso inadecuado $u$ omisión de la puntuación y acentuación. Por otro lado, se muestran problemas de estilo que comprometen la precisión de información en el texto o bien su adecuación al género, influyendo, incluso, en aspectos de su macroestructura. A continuación, se señalan y ejemplifican los problemas más evidentes dentro de las referidas categorías.

Se observa que las dificultades más recurrentes en la microestructura textual se ubican en la subcategoría puntuación y acentuación. Del total de las dificultades de microestructura, un $32.1 \%$ corresponde a problemas en el uso de la coma. A ese respecto se menciona, a modo de ejemplo, la omisión o empleo inadecuado. A continuación, se presenta uno de los casos más identificados como problemas de omisión de la coma.

- La reforma de los planes de estudio se basó, entre otras cuestiones en la necesidad de fortalecer y potenciar el perfil de egresado... (T6)

El fragmento previo muestra ausencia de la coma, pues su uso es requerido para separar expresiones aclaratorias/explicativas dentro de la enunciación. En este caso, "entre otras cuestiones" es una expresión aclaratoria.

El siguiente ejemplo se caracteriza por el empleo inadecuado de la coma. La inadecuación, en este contexto, está en el uso de la coma entre sujeto y predicado.

- Finalmente, se concluye que el celular como mediador educativo, tiene una serie de ventajas/desventajas y de "mitos"...(T1)
Otro inconveniente que concurre con la subcategoría coma respecta al uso de la tilde y representa un $20.7 \%$ de los problemas con microestructura. Se configura por la omisión o por su empleo inadecuado. Se muestran algunos casos de omisión de la tilde.

- ... permiten analizar que dicen los estudiantes acerca de Enfermería como profesión... (T2)

- Estos fundamentos nos hacen pensar como son las prácticas de enseñanza... (T7)

- Se viene observando un incremento sostenido en el numero de ingresantes... (T10)

- Se debe promover que la salud mental este a cargo de un equipo interdisciplinario... (T5)

- ... la tasa bruta de ingresante en los últimos 10 años se multiplico por 4... (T10)

Las omisiones de la acentuación gráfica identificadas en los textos se revelan en pronombres o adverbios interrogativos, vocabulario y tiempos verbales que exigen el empleo de la tilde. Su ausencia en los ejemplos señalados compromete el significado de las palabras e, incluso, puede perjudicar el sentido de la oración.

Si por una parte se identifica la falta de la tilde, por otra se advierte su uso inapropiado:

- ... esta investigación se proyecta desde el paradigma cualitativo, dónde instrumentos tales como encuestas y entrevistas... (T4)

- Existe profusa bibliografía que dá cuenta de los beneficios... (T10)

- ... incluyendo también el tipo de exámen utilizado... (T13)

Una de las inadecuaciones a ese respecto está en el empleo de la tilde en adverbios relativos (como el adverbio relativo de lugar donde). Este equívoco se da por la confusión con el adverbio interrogativo dónde. Tal como ocurre con la omisión de la tilde, su empleo inadecuado, en este caso, también puede comprometer la semántica oracional. 
Las inadecuaciones de acento gráfico recaen, aún, en algunos monosílabos tónicos y en sustantivos en singular que deben ser acentuados solo en plural. Respectivamente, los ejemplos nos muestran "da" y "examen" acentuados inapropiadamente. El uso de la tilde en "da" podría atribuirse a la hipergeneralización de la regla de otros monosílabos que llevan tilde para diferenciar el significado.

Aun dentro del ámbito puntuación y acentuación, los problemas se revelan también en lo relativo a dos puntos y a punto y coma. El primero se caracteriza por la omisión, mientras que el segundo se constata en su ausencia o empleo inadecuado. Teniendo en cuenta, pues, las dificultades de microestructura, la subcategoría dos puntos denota un $7.9 \%$, mientras que los problemas correspondientes a punto y coma apuntan al 5\%.

Se presentan algunos ejemplos de omisión de dos puntos identificados en los textos.

- Se analizará el Perfil del Graduado en relación con las actividades reservadas a las dos titulaciones, Enfermero y Licenciado en Enfermería. (T2)

- La información se presenta sistematizada en dos cuerpos principales, uno que refiere a concepciones epistemológicas y otro, a las estrategias didácticas. (T4)

- ... hay diferentes factores que influyen en el rendimiento académico; las condiciones ambientales, las aptitudes, los intereses, la personalidad, los hábitos de estudio, la motivación, ... (T10)

El no uso de dos puntos, según apuntan los ejemplos, se caracteriza por la aplicación de otros signos de puntuación en su lugar. En ese sentido, existen construcciones sintácticas en que los dos puntos son fundamentales. Frases que mencionan enumeraciones son ejemplos de la importancia funcional de este signo. No obstante, en los textos se identifican registros de enumeraciones antecedidos por coma o punto y coma. Teniendo en cuenta ese uso enmascarado de los dos puntos, es decir, recurriendo a otros signos (pero que no lo sustituyen), es que se denomina tal ocurrencia como omisión de dos puntos.

En cuanto al punto y coma, se exponen primeramente las ocurrencias relativas a la ausencia de este signo.

- Podemos decir entonces que no genera soluciones sino que entrena al estudiante para generarlas, además al poder implementarse en grupos... (T11)

- La organización académica-administrativa la de carrera de MV depende de la FB, por consiguiente, adecua sus actos académicos al Reglamento Académico General de la... (T13)

El uso de punto y coma se establece por una pausa mayor entre las oraciones. En los fragmentos previos es posible notar, por el contexto de las frases, un intervalo mayor entre ellas. No obstante, este lapso está demarcado por la coma. Aunque esta representa un signo de puntuación muy recurrente en producciones textuales, no sustituye al punto y coma.

Si bien hay casos en los que figura la ausencia del punto y coma, hay también otros en que se muestra el empleo inadecuado del punto y coma:

- La idea de trabajar bajo este interrogante surgió luego de observar distintas clases en las que los estudiantes aumentaban su participación ante la presencia de este dispositivo; a raíz de esto se comenzó con la lectura de distintos textos que profundizaban este tema, muchos de los cuales... (T1)

- Este modelo rompe con la metodología tradicional de la asignatura donde el profesor expone la temática y los alumnos la estudian en su casa; con el modelo Flipped Classroom los estudiantes llegan a la clase con conocimientos de los temas que... (T10)

- Desde que comenzó la UDA; los estudiantes han interrogado y reclamado sobre... (T5)

Los fragmentos arriba indicados ejemplifican las dificultades en lo que atañe al uso del punto y 
coma. Su empleo figura, principalmente, en lugares que requerirían el punto y seguido o la coma.

En lo concerniente al estilo, se identifican tres tipos de dificultades más representativas. A partir de ello, se establecen las siguientes subcategorías: empleo de lenguaje coloquial, omisión de conectores oracionales y oraciones truncas. De las tres subcategorías, la primera representa un 7.1\% de las dificultades de microestructura, al tiempo que las dos últimas ocurren en menor grado de frecuencia y, en igual representatividad, corresponden a un $4.3 \%$ cada una. Se evidencia, pues, que las ocurrencias en la categoría estilo son menos frecuentes que las relativas a la puntuación y acentuación. Los siguientes ejemplos muestran el empleo de lenguaje coloquial:

- Por dar un ejemplo, en 2018 hubo 3.208 inscriptos. (T3)

- Por lo que, el desarrollo de la misma fue un gran desafío para los docentes tutores. (T5)

- Dicho proyecto se centra en el análisis de las estrategias de... (T7)

En el análisis de los textos se identifican algunas expresiones que no responden adecuadamente a las exigencias lingüísticas del uso de un lenguaje formal del género textual plan de tesis. Por lo tanto, expresiones como Por dar un ejemplo y Por lo que requieren su sustitución por un lenguaje más culto. Respectivamente podrían ser reemplazadas por: A modo de ejemplo y Por esta razón.

En cuanto a la omisión de conectores oracionales, a continuación, se ilustran algunos ejemplos $\mathrm{y}$, luego, se expresan algunas consideraciones.

- ... se los comparará con los resultados de las clases en las [*] este dispositivo tecnológico no estuvo presente. (T1)

- El presente estudio se llevará a cabo con un abordaje de tipo político institucional debido $\left[{ }^{*}\right]$ que la problemática de abandono se analizará en un contexto... (T3)

- ... se han llevado cabo propuestas metodológicas a los fines [*] acercar al estudiante conceptos centrales... (T4)
En los fragmentos previos se evidencia la falta de los conectores que, a, de, respectivamente. Aunque su ausencia pueda ser inferida por el lector, es un tema que merece atención puesto que esta laguna es una de las causas de interferencia en la fluidez de la lectura. Consecuentemente también implica en la calidad del texto. Una revisión atenta de la escritura puede evitar inconvenientes de esta propiedad.

Análogamente a la problemática omisión de conectores oracionales se establece la categoría de oraciones truncas. Aunque las dos se ocupan de la estructura de las frases, son consideradas por separado ya que la primera contempla específicamente los conectores en las frases, mientras que la segunda considera problemas estructurales que dificultan la claridad de las informaciones dentro de la frase. Así, su atención se decanta hacia la sintaxis y también a la semántica, si bien, por tratarse de una categoría de problemas microestructurales, los aspectos semánticos son considerados solo a nivel oracional y no textual1. A continuación ${ }^{1}$, se ejemplifican algunas oraciones truncas.

- Este proyecto se llevara (sic) a cabo mediante un estudio descriptivotransversal, el cual buscara (sic) recoger [*] (T7)

- [*] Para responder a la pregunta. ¿Las prácticas hospitalarias representan los objetivos que se plantean en el plan de estudios? (T5)

- La distancia entre las universidades que tenemos y la agenda [*] Así como escribir es un proceso, desarrollar casas de altos estudios más inclusivas tampoco es un estado que se tiene o no se tiene indefectiblemente sino una de las metas de un país democrático. (T7)

\footnotetext{
${ }^{1}$ La semántica textual es parte de la macroestructura; por ende, no debe ser confundida con la semántica oracional, que es aquella que considera el sentido únicamente de la frase y, de ser así, se enmarca en la microestructura.
} 
Las oraciones truncas se caracterizan por ser frases incompletas que, por esa razón, denotan sentido impreciso y/o confuso del enunciado. Al igual que la omisión de conectores oracionales, también respecto a las oraciones truncas se hace imprescindible la revisión textual.

Las dificultades tratadas en la microestructura se relacionan con el giro comunicativo promovido en Argentina por la Reforma Educativa de 1994, cuya principal característica en el campo de la enseñanza de lengua fue reemplazar la centralidad de la gramática estructural oracional por la gramática textual, con énfasis en el plano discursivo de uso de la lengua. En los últimos años, se ha observado en estudios no solo nacionales sino también internacionales (Zárate Fabián, 2017) que los estudiantes universitarios siguen teniendo dificultades del orden de la morfosintáctica oracional en su escritura académica, lo que pone de relieve la necesidad de volver a otorgar centralidad a la enseñanza de la gramática. Esta centralidad, sin embargo, se reivindica no en los moldes mecánicos de enseñanza de gramática como explicación-ejercitación, sino en una reflexión gramatical que retome contenidos morfosintácticos en articulación con la reflexión discursiva, al inicio de la formación universitaria (Schere, 2020).

\section{Dificultades de macroestructura}

Como se ha mencionado, la macroestructura se relaciona directamente con la cohesión global de los temas tratados en un texto. De ese modo, para el análisis, se rescatan dos tipos de dificultades que aparecen en la totalidad de los textos, como se observa en la Tabla 1. Estas son, por un lado, a las dificultades para relacionar ideas, comprendidas en el ítem Incoherencia entre ideas y/u objetivos (y entre párrafos) y, por el otro, a la Información incompleta o imprecisa. Además, por la proximidad temática, también se incorpora al análisis la falta de cohesión entre el cierre de una sección e inicio de otra o entre párrafos. Estos tres ítems suman más de la mitad (55\%) de las dificultades encontradas en la macroestructura. Estos problemas fueron los más citados por estudiantes encuestados en el marco de una investigación sobre la apropiación del discurso académico por parte de jóvenes universitarios en la Provincia de
Córdoba, Argentina (Borioli, 2019). En este aspecto, los estudiantes mencionaron tener dificultades para producir cohesión entre las ideas, para articular los contenidos y los autores, y para usar los conectores. En nuestro corpus también se observan estos tipos de problemas, como se aprecia en los próximos párrafos.

En cuanto a la materialidad discursiva, las dificultades para relacionar ideas se presentaron de tres modos predominantes: entre lo que se plantea y los objetivos propuestos; entre párrafos; y entre el cierre de una sección e inicio de otra. Para ilustrar ese tipo de dificultades se toman como ejemplo la primera sección del T8, llamada Justificación. Esta sección se desarrolla a lo largo de dos páginas que suman 19 párrafos y, aunque no exista una cantidad de párrafos esperada para cada sección, se observa una exageración de formulaciones muy cortas que no están encadenadas temáticamente. En este sentido, se destacan dos proposiciones cortas y que no llevan conectores que las pueda relacionar y otorgar fluidez al escrito:

\section{- Con todas estas etapas evaluativas aprobadas, el alumno regulariza la materia. \\ - [*] Año a año la cantidad de alumnos que alcanzan la regularidad es de aproximadamente el $90 \%$ y el 10\% que no logra la regularidad, en su mayoría corresponde a alumnos que han abandonado el cursado o no aprobaron la instancia de evaluación MC on-line. (T8)}

El primer párrafo en el ejemplo previo está constituido, inadecuadamente, por una única frase. Este fragmento podría venir en el cierre de su párrafo precursor, donde se enumeran las etapas que los estudiantes en cuestión deben cumplimentar para regularizar una materia. Asimismo, además de los problemas de orden microestructural, el segundo párrafo del fragmento seleccionado carece de un conector inicial que relacione las ideas anteriores con los datos estadísticos.

En el Texto 12 se selecciona un fragmento que también corresponde a un párrafo corto y con una articulación débil con los párrafos precedentes. 
- Según esto, los alumnos que acrediten la totalidad de las asignaturas aprobadas de la carrera obtendrán como título el de Médico Veterinario Generalista. (T12)

En este caso, como se trata de un párrafo aparte, se observa la necesidad de recuperar el antecedente del deíctico esto para de entender completamente el sentido de la idea. Se podría adecuarlo a través de la reposición señalada, por ejemplo: Según esta organización, los alumnos (...).

Como ejemplo de una dificultad de articulación entre el cierre de una sección e inicio de otra, se presenta un fragmento del Texto 10, correspondiente al último párrafo de un apartado denominado Antecedentes, previo a los Objetivos:

\section{- Además Broglia (2011) hace referencia al impacto que este trabajo tendrá en los docentes, ya que se generará una experiencia nueva que fomenta el trabajo grupal e interdisciplinario y el contacto directo con el caso clínico 'sobre la camilla'. (T10)}

En este caso, la sección podría terminar con un cierre que articule lo que ya se ha estudiado y lo que está pendiente, para justificar lo que el cursante propone como objetivos de su tesis.

A continuación, se describe otra dificultad macroestructural que también se presentó en la totalidad de los textos analizados: Información incompleta o imprecisa. Esta categoría remite a recurrir a estudios previos respecto a un tema, sin indicar la fuente de información:

\section{- Así es como muchos ensayos destacan las contundentes cualidades del estudio de casos o la resolución de problemas mientras que otros abordan la implementación de las nuevas tecnologías. (T4)}

En ese fragmento se observa la nulidad de referencia concreta a qué ensayos y a qué textos indica el autor-escritor.
Como se refiere en la introducción de este artículo, el texto académico se caracteriza por incorporar tanto las voces de autores especialistas en el tema, como la propia voz de quien escribe. Esta articulación de voces se percibe en la literatura sobre el tema como uno de los grandes desafíos en la escritura en el posgrado (Borioli, 2019; Castro Azuara \& Sánchez Camargo, 2016; Giraldo-Giraldo, 2020; Nishida, 2020; Rodríguez Hernández \& García Valero; 2015). Las dificultades refieren a falta de conocimiento del formato de citación y dificultad para construir un discurso propio que dé cuenta de la voz del autor y, al mismo tiempo, de un diálogo con los autores revisados.

En el inicio del apartado Metodología del Texto 10 se visualiza información incompleta:

\section{- Metodología cualitativa, interpretativa o etnográfica, debido a que utiliza la recolección de datos no numérica para descubrir o afinar preguntas de investigación en el proceso de interpretación (Sampieri, 2008, p 8). Estudio longitudinal (estudio de seguimiento de un grupo de sujetos). (T10).}

La redacción se presenta de forma telegráfica y, por lo tanto, no articulada hacia dentro ni tampoco hacia el conjunto del apartado. En la última frase, no se ha construido una proposición verbal. En este caso, diferente del fragmento del Texto 4 mencionado anteriormente, el cursante se ha respaldado en una referencia bibliográfica. Pese a esto, la forma telegráfica y paraverbal denuncia la falta de elaboración propia respecto a lo que cita, vaciando la potencialidad del recurso argumentativo de citar fuentes.

Se entiende que la práctica de recurrir a argumentos sin respaldo científico, o bien recurrir débilmente a las fuentes, es común en lo que atañe a los géneros discursivos orales que circulan en los ambientes laborales, sobre todo entre docentes refiriéndose a lo que ocurre, empero, sin citar las fuentes. El trabajo académico plasmado en la escritura implica también, justamente, testear los consensos en los grupos profesionales y buscar respaldo científico que los avalen. En este sen- 
tido, con que se citara algunos autores y fechas, el fragmento seleccionado del Texto 4 quedaría más adecuado.

\section{Dificultades de superestructura}

Finalmente, en la tercera gran categoría, dificultades de superestructura, se señalan las inadecuaciones relativas al género textual académico plan de tesis. De ese modo, en la problemática de la estandarización genérica, se discuten sus puntos más destacados: el cronograma, las citas en el texto y las referencias; la suma de estas categorías representa el $56 \%$ de las dificultades de Superestructura. Estos tres componentes le otorgan gran parte de la identidad de género a la arquitectura interna del plan de tesis.

La academia supone la circulación de textos escritos con una estética uniforme, en la que prevalece un mismo tipo de letra, un margen justificado, letras de color negro, el uso de negritas solo en los títulos entre otros aspectos. Sin embargo, los cursantes no necesariamente cuentan con la apropiación de estas formas requeridas a la hora de producir sus propios textos. Esto coincide con los hallazgos de Arroyo González, \& Gutierrez Braojos (2016), quienes señalan carencias en el avance en los procesos de escritura a lo largo de la carrera universitaria.

Estos aspectos circundan el contexto formativo en que los docentes advierten la necesidad de la enseñanza de la escritura durante el dictado de Talleres de tesis, cuyo foco primordial radica en la práctica de estrategias de investigación en paralelo a la enseñanza de estrategias lingüísticas propias del ámbito académico. Queda patente que no hay contenido sin forma.

En este sentido, Di Stefano y Pereira (2012) refieren a la materialidad gráfica, natural en el ámbito académico, que sin embargo, frecuentemente se presenta como ajena a sus integrantes, particularmente entre estudiantes, incluso de posgrado. En este sentido, el cronograma de un plan de tesis, pese a que se pueda presentar de maneras muy singulares, mantiene componentes fijos, relacionados a las etapas de una investigación. De este tipo de producción textual se espera una enumeración de actividades secuenciadas temporalmente que describan genéricamente cada paso necesario de la investigación. Estos pasos son confrontados en su eje opuesto con la temporalidad que ocupará el desarrollo proyectado.

Ahora bien, en el corpus se identifica que, del total de textos analizados, ocho tuvieron algún tipo de problema en la producción del cronograma. Incluso, uno de los sujetos no presentó este apartado. A su vez, las otras dificultades dentro de esta subcategoría se relacionan a la Incoherencia en el orden de las actividades propuestas, significando el $7.5 \%$ del total de dificultades de superestructura. Se considera un valor representativo, puesto que se ubica detrás solo de los problemas inherentes a las referencias. Se presentan ejemplos seleccionados de los casos más paradigmáticos.

El Texto 2 consigna 13 actividades en su cronograma. Entre ellos, algunas estaban sobrepuestas: "Relevamiento y análisis de documentación pertinente al objeto de investigación”, "Analizar investigaciones precedentes sobres los ejes temáticos de la investigación" y "Relevamiento bibliográfico inicial, exploración y análisis de los datos recogidos por Internet". El ítem número cinco del cronograma preveía "Entrevistas a informantes claves", mientras que en el ocho se proponía la "Prueba piloto de la entrevista" y recién en el ítem nueve "La construcción del marco referencial". Este caso da cuenta de la falta de coherencia temporal entre los pasos a seguir en la investigación. Aquí, la estructura formal de presentación indica dificultades de comprensión del proceso de una investigación. En menor medida, las mismas dificultades aparecieron en otros textos del corpus.

Con relación a las Referencias, se conciben como la estandarización predeterminada por las normas de redacción académica utilizado en la Universidad en cuestión: autores, obras, documentos, sitios web, entre otros. Desde esta perspectiva, los problemas emergen de la ausencia de referencias (o parte de ellas) a lo largo del texto, como: autor, año o página. Un $21.7 \%$ de las dificultades de superestructura son representadas por esta categoría.

Igualmente, en la estandarización de las referencias, en el último apartado del texto, protagonizan los otros $21.7 \%$ de las dificultades de superestructura. En ese sentido, se observan dife- 
rentes inconsistencias: mixtura entre las normas APA y Vancouver, información fuera de lugar, información incompleta, mención a textos no citados en el cuerpo del plan de tesis u omisión de textos citados, falta de orden alfabético y, en uno de los casos, ausencia completa de este apartado.

En este sentido, Núñez Cortés \& Errázuriz Cruz, 2020 señalan un desconocimiento, por parte de los estudiantes, de las convenciones discursivas propias del ambiente universitario antes de comenzar sus estudios superiores, y a lo largo de estos. Por otra parte, Giraldo-Giraldo (2020) advirtió que más del $80 \%$ de estudiantes colombianos de posgraduación encuestados sobre sus dificultades de escritura expresaron conocer las normas de escritura académica. Esto indica que el reconocimiento de los criterios propios de géneros discursivos académicos no es garantía de su correcta aplicación. En otras palabras, las dificultades notificadas en los diferentes niveles de escritura no implican necesariamente falta de conciencia sobre las reglas, sino más bien una distancia entre el conocer y el aplicar.

En ese sentido, el paso por la universidad no garantiza la evolución en la competencia escritora (Arroyo González \& Gutierrez Braojos, 2016). Como afirma Navarro (2019, p. 26), "lamentablemente, los géneros discursivos de formación suelen ser adquiridos mediante prácticas espontáneas de enculturación y muy pocas veces explicitados con fines de enseñanza o, menos aún, curricularizados". Esto refuerza la necesidad de contemplar la enseñanza de la escritura académica en la universidad explicitada en el currículo, partiendo desde la carrera de grado y "de manera transversal en la impartición de todas las asignaturas" (Coronado-Hijón, 2017, p. 28).

Partiendo de lo anterior, la superestructura de un texto funciona como un primer acercamiento que permite advertir problemas de base con respecto a los contenidos relacionados a la formación misma del espíritu científico. A su vez, contextualiza el análisis descendente para llegar a la macro y microestructura textual y proyectar las estrategias más adecuadas para subsanar estas dificultades.

\section{Consideraciones finales}

En este artículo se han analizado las producciones finales de los estudiantes de posgrado en torno al plan de Tesis. En esta instancia, si bien las faltas relacionadas a la microestructura, en su sentido gramatical (sintaxis, coherencia entre frases, puntuación, entre otros), podrían ser resueltas por un revisor de textos, por ejemplo, los problemas relacionados a la adecuación genérica textual son de resolución más compleja, ya que se refieren a la acción investigativa en sí misma.

Las dificultades que están relacionadas con la adecuación al género textual plan de tesis apuntan a una interrogante pertinente para conducir las preocupaciones de los docentes abocados a la enseñanza de la escritura académica en instancias de posgrado. Al respecto, Navarro (2019) propone una "didáctica basada en géneros» que consiste en la enseñanza explícita e intencionada de la lectura, la escritura y la oralidad académicas. Esta permite que los estudiantes provenientes de sectores sociales desventajados puedan tener las mismas oportunidades que los pertenecientes a familias con mayor capital cultural y, en particular, semiótico.

Es necesario indicar que cada uno de los tres grandes niveles que se han tomado de Van Djik (1980) para orientar la clasificación de dificultades se superponen, como el mismo autor lo explicita. Es decir, en el análisis de un cronograma se denotan aspectos microestructurales, como errores ortográficos o sintácticos, y también problemas macroestructurales, como los de conexión entre un ítem del cronograma y otro. Más allá de esa interconexión, distribuirlos de ese modo resulta fructífero para focalizar cada dificultad por vez.

Como lo ha demostrado la literatura sobre el tema, las carreras humanísticas tienen muchas más prácticas de escritura que las carreras de la salud o de las ciencias exactas. En el presente caso, se cuenta con una sola participante proveniente del campo humanístico. No se puede, por eso, arrojar conclusiones al respecto, pero sí se rescata lo expresado por Moreno et al. (2009, p. 1): “a escribir [bien] se aprende escribiendo". Un futuro estudio podría cotejar la perspectiva del 
docente con la de los estudiantes acerca de las dificultades en la escritura del Plan de tesis.

\section{Agradecimientos}

El presente trabajo forma parte de un Proyecto mayor en curso denominado "Escritura de tesis de posgrado", subsidiado por la Agencia Nacional de Promoción Científica y Técnica de Argentina.

\section{Referencias}

Arnoux, E. (dir.) (2009). Escritura y producción de conocimiento en posgrado. Buenos Aires: Santiago Arcos.

Arroyo González, R., \& Gutierrez Braojos, C. (2016). Competencias escritoras en la formación universitaria del profesorado. Revista Electrónica Interuniversitaria de Formación del Profesorado, 19(3), 135-147. http:// doi.org/10.6018/reifop.19.3.221191

Bajtín, M. (2002). Estética de la creación verbal. Buenos Aires: Siglo XXI. https://circulosemiotico.files. wordpress.com/2012/10/estetica-de-la-creacic3b3n-verbal.pdf

Borioli, G. (2019). "Me cuesta todo". Escritura académica y educación superior. Investiga+, 2(2), 1-14. http:// www.upc.edu.ar/wp-content/uploads/2015/09/investiga_mas_a2n2.pdf

Bronckart, J.-P. (2004). Actividad verbal, textos y discursos. Por un interaccionismo socio-discursivo. Madrid: Astáviz Fotomecánica.

Carlino, P. (2013). Alfabetización académica diez años después. Revista Mexicana de Investigación, 18(57), 355-381. https://www.redalyc.org/pdf/140/14025774003.pdf

Cassany, D. (2009). La composición escrita en E/LE. Revista Marco ELE, 9, 47-66. https://marcoele.com/descargas/expolingua_1999.cassany.pdf

Castro Azuara, M. C. \& Sánchez Camargo, M. (2016). La formación de investigadores en el área de humanidades: Los retos de la construcción de la voz autoral en la escritura de la tesis de doctorado. Revista signos, 49(1), 30-51. doi: 10.4067/S071809342016000400003

Chois, P. \& Jaramillo, L. (2016). La investigación sobre la escritura en posgrado: estado del arte. Lenguaje, 44(2), 227-259. http://www.scielo.org.co/pdf/leng/ $\mathrm{v} 44 \mathrm{n} 2 / \mathrm{v} 44 \mathrm{n} 2 \mathrm{a} 05 . \mathrm{pdf}$

Coronado-Hijón, A. (2017). Escritura académica: retos y enfoques ante sus dificultades de aprendizaje. Magíster: Revista miscelánea de investigación, 29(1), 25-30. https://dialnet.unirioja.es/servlet/articulo?codigo $=6493823$

Di Stefano, M. \& Pereira, M. C. (2012). Concepción sociodiscursiva de la lectura y la escritura y su enseñanza.
Actas del IV Congreso Internacional de Investigación y Práctica Profesional en Psicología. Buenos Aires: UBA. http://www.semiologia-cbc-distefano. com.ar/publicaciones/articulos/di-Stefano-Pereira-Ponencia-IV-Congreso-Psicologia.pdf

Eco, U. (1998). Cómo se hace una tesis. Barcelona: Gedisa.

Fuenmayor, G., Villasmil, Y. \& Rincón, M. A. (2008). Construcción de la microestructura y macroestructura semántica en textos expositivos producidos por estudiantes universitarios de LUZ. Letras, 50(77), 189-219. http://ve.scielo.org/scielo.php?script=sci arttext\&pid=S0459-12832008000200007

Gioffredo, R. y Pozzo, M.I. (2015). Diseño e implementación de proyectos en la formación de posgrado. La propuesta de la Especialización y Maestría en Docencia Universitaria de la UTN, Facultad Regional Rosario. Actas del III Congreso Nacional y Latinoamericano de la Red Argentina de Posgrados en Educación Superior (REDAPES).

Giraldo-Giraldo, C. (2020). Dificultades de la escritura y desaprovechamiento de su potencial epistémico en estudiantes de posgrado. Revista Colombiana de Educación, 1(80), 173-192. doi: 10.17227/rce.num80-9633

Ilich, M. E. \& Morales, O. A. (2004). Análisis de textos expositivos producidos por estudiantes universitarios desde la perspectiva lingüística discursiva. Educere, 8(26), 333-345. https://www.redalyc.org/ pdf/356/35602607.pdf

Klein, I. (coord.). (2007). El taller del escritor universitario. Buenos Aires: Prometeo.

Mendoza Ramos, A. (2014). Las prácticas de evaluación docente y las habilidades de escritura requeridas en el nivel posgrado. Innovación Educativa, 14(66), 147-176.

Moreno, C., Zurita, P., \& Moreno, V. (2009). A escribir se aprende escribiendo. Revista MarcoELE (8). https:// marcoele.com/a-escribir-se-aprende-escribiendo/

Navarro, F. (2019). Aportes para una didáctica de la escritura académica basada en géneros discursivos. DELTA: Documentação de Estudos em Lingüística Teórica e Aplicada, 35(2), 1-32. doi: 10.1590/1678$460 \times 2019350201$

Nishida, F. (2020). Problemáticas en las prácticas de lectura y escritura de los estudiantes de posgrado. Trabajo Final Integrador (Especialización en Docencia Universitaria). Universidad Nacional de La Plata. http:// sedici.unlp.edu.ar/handle/10915/108438

Núñez Cortés, J. A., \& Errázuriz Cruz, M. C. (2020). Panoramas de la alfabetización académica en el ámbito iberoamericano: aportes para la calidad de la Educación Superior. Tendencias Pedagógicas, 36, 1-8. doi: 10.15366/tp2020.36.01

Pozzo, M.I. (2019). Incidencia de las trayectorias disciplinares en la escritura en el posgrado: la perspectiva de los estudiantes. Revista Linguagem \& Ensino, (22) 3, 809-834. Universidad Federal de Pelotas. DOI: HTTP://DX.DOI.ORG/10.15210/RLE.V22I3.16676. 
Pozzo M. I. (2020). Escritura de tesis de posgrado. Desde el proyecto hasta la defensa. Buenos Aires: Biblos.

Rodríguez Hernández, B. A., \& García Valero, L. B. (2015). Escritura de textos académicos: dificultades experimentadas por escritores noveles y sugerencias de apoyo. CPU-E, Revista de Investigación Educativa, 20, 249-265. https://www.redalyc.org/articulo. oa?id=2831/283133746012

Rueda, N. (2007). Lectura comprensiva: procesos cognitivos y tipos de texto. En G. Biber (comp.). La lectura en los primeros años de la universidad. Córdoba: Educando ediciones.

Schere, J. (2020). Escritura académica y reflexión gramatical en el comienzo de la formación universitaria. Álabe, 22, 1-15. doi: 10.15645/Alabe2020.22.7
Stake, R. (1998). Investigación con estudio de casos. Madrid: Morata.

Swales, J. (1990). The Concept of Discourse Community. En J. Swales, Genre Analysis: English in Academic and Research Settings (pp. 21-32). Boston: Cambridge University Press.

Swales, J. (2004). Research Genres. Explorations and Applications. Glasgow: Cambridge University Press.

Van Dijk, T. A. (1980). Estructuras y funciones del discurso. Barcelona: Siglo XXI.

ZárateFabián, M. C. (2017). La escritura académica:Dificultades y necesidades en educación superior. Educación Superior, 2(1), 46-54. http://www.scielo.org.bo/scielo.php?script=sci_arttext\&pid=S2518-82832017000100005\&lng=es\&tlng=es

RIDU / Revista Digital de Investigación en Docencia Universitaria / e-ISSN: 2223-2516

(c) Los autores. Este artículo es publicado por la Revista Digital de Investigación en Docencia Universitaria del Área de Institutional Research and Effectiveness de la Dirección de Aseguramiento de la Calidad, Universidad Peruana de Ciencias Aplicadas. . "Este es un artículo de acceso abierto, distribuido bajo los términos de la LicenciaCreativeCommons Atribución 4.0 Internacional (CC BY 4.0) (https://creativecommons.org/licenses/by/4.0/deed.es), que permite el uso, distribución y reproducción en cualquier medio, siempre que la obra original sea debidamente citada." 